\title{
Transport Properties with Gauss-Bonnet corrections using soft-wall model of holographic QCD
}

\author{
Neha Bhatnagar \\ Department of Physics, Banaras Hindu University(BHU), Varanasi-05, India \\ E-mail: bhtngr.neha@gmail.com
}

\begin{abstract}
We study the renormalization group (RG) flow of transport coefficients of a $3+1$ dimensional strongly coupled system using holography. Introducing Gauss-Bonnet corrections in the gravity system we study the behavior of ac conductivity and diffusion constant using the soft-wall model of the bottom-up approach. Further we show the dependence of transport coefficients on the chemical potential of the system.
\end{abstract}

Keywords: Holographic QCD, soft-wall model, Gauss-Bonnet corrections 


\section{Introduction}

AdS/CFT duality (holography) is a remarkable tool to study the dynamics of strongy coupled system. This duality was first introduced by Maldecena [1] showing a correspondence between $\mathscr{N}=4$ super Yang-Mills gauge theory in 4 dimensions with type II B string theory in $A d S_{5} \times S_{5}$ bulk space. Later research and discussions generalised this conjecture to study strongly coupled systems in other branch of physics like condensed matter physics, quark gluon plasma (QGP) and cosmology using their weakly coupled dual gravitational system in the large $\mathrm{N}$ limit approximation.[2, 3, 4, 5]

Using holography we work in the soft-wall model and study the behavior of transport coefficients of a 3+1 dimensional system using its dual Einstein-Maxwell system in 4+1 dimensions with Gauss-Bonnet (GB) corrections. Soft-wall model [6] is a modified form of hard model with a dilaton field (to introduce the confinement) has successfully depicts the hadron spectra and Regge trajectory. Applying the soft-wall model for this system we calculate the RG flow of ac conductivity with varying chemical potential in the probe limit. Frequency dependence of ac conductivity and the dependence of diffusion constant on GB term has also been shown.

\section{Holographic Model and Results}

Einstein-Maxwell system with Gauss-Bonnet $\left(R_{G B}\right)$ corrections in the soft wall model is given,

$$
S=\int d^{5} x \sqrt{-g} e^{-\phi}\left\{\frac{1}{2 \kappa^{2}}\left(R-2 \Lambda+\lambda R_{G B}\right)-\frac{1}{4 g^{2}} F^{2}\right\},
$$

where $\lambda$ is the Gauss Bonnet Coupling constant and $R_{G B}=R^{2}-4 R_{M N} R^{M N}+R^{M N P Q} R_{M N P Q} . F^{2}$ is the gauge field strength with $F_{\mu \nu}=\partial_{\mu} A_{\nu}-\partial_{v} A_{\mu}$. Using $l=1$ for calculations with cosmological constant $\Lambda=\frac{-6}{l^{2}}$, the charged black hole solution of the above system is given as,

$$
d s^{2}=\frac{r_{+}^{2}}{l^{2} u}\left(-f(u) N^{2} d t^{2}+\sum_{i=1}^{3} d x^{i} d x^{i}\right)+\frac{l^{2} d u^{2}}{4 u^{2} f}
$$

where $N^{2}=\frac{1}{2}(\sqrt{1-4 \lambda}+1), f(u)=\frac{1}{2 \lambda}\left(1-\sqrt{1-4 \lambda(1-u)\left(1+u-a u^{2}\right)}\right.$, with $a=\frac{l^{2} \kappa^{2} Q^{2}}{6 g^{2}}$, where charge $(\mathrm{Q})$ and chemical potential $(\mu)$ of the black hole is given as $Q=\frac{2 \mu}{r_{+}}$. Thus we can write equation of motion as,

$$
\begin{array}{r}
R_{\mu v}+\frac{1}{2} g_{\mu v} R-\Lambda g_{\mu v}=8 \pi T_{\mu v} \\
\nabla_{\mu} e^{-\phi} F^{\mu v}=0
\end{array}
$$

taking the linear dilaton profile $\phi=c u$ as in [7], with $c=0.388 \mathrm{GeV}^{2}$. Introducing the metric and gauge field perturbations (taking $h_{u n}=0$ and $A_{u}=0$ as the gauge condition), $g_{m n}=\mathbf{g}_{\mathbf{m n}}^{\mathbf{0}}+e^{\phi} \tilde{h}_{m n}$ and $A_{m}=\mathbf{A}_{\mathbf{m}}+\tilde{A}_{m}$.

The Fourier transform of the fields is given as,

$$
\begin{aligned}
\tilde{h}_{m n}(t, z, u) & =\int \frac{d^{4} k}{(2 \pi)^{4}} e^{-i \omega t+i k z} h_{m n}(k, u) \\
\tilde{A}_{m}(t, z, u) & =\int \frac{d^{4} k}{(2 \pi)^{4}} e^{-i \omega t+i k z} A_{m}(k, u),
\end{aligned}
$$




\subsection{AC conductivity}

AC conductivity can be calculated using the linearised equation of motion for the gauge field.

$$
A_{x}^{\prime \prime}+\left(\frac{f^{\prime}}{f}-c\right) A_{x}^{\prime}+\frac{l^{4}}{4 N^{2} f^{2} r_{+}^{2} u}\left(\omega^{2}-k^{2} f\right) A_{x}-e^{\phi} \frac{1}{N^{2} f} h_{t}^{\prime x}=0
$$

Using gauge field equation of motion we get the frequency dependence of ac conductivity as shown in Fig. 1 where the real part of the conductivity shows Drude peak.
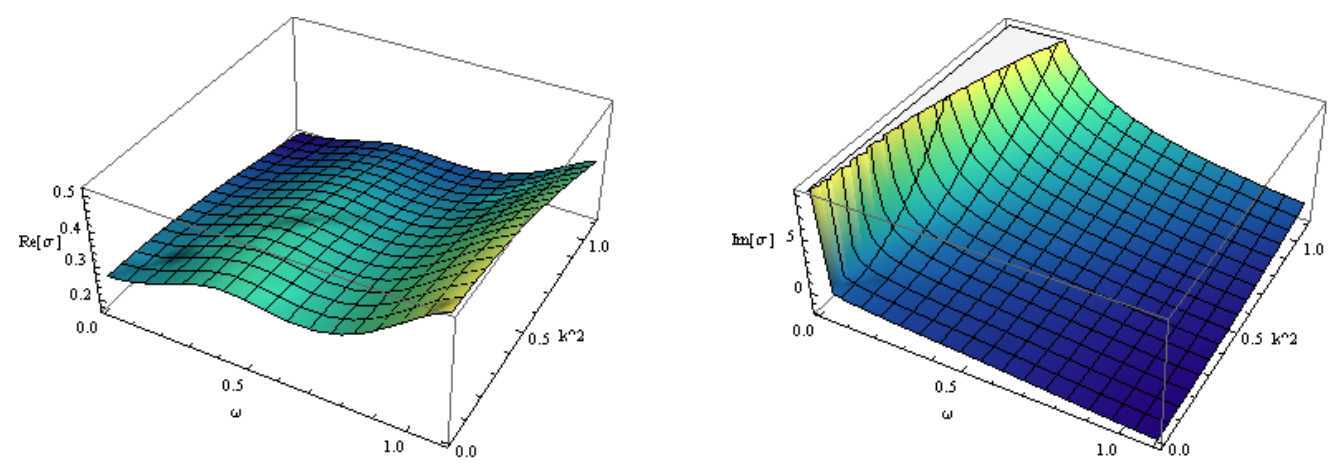

Figure 1: Frequency and momentum dependence of ac conductivity at $\lambda=0.01$ for $\mu=0.05$

Now at zero momentum limit we calculate the ac conductivity flow at frequency $\omega=0.9$ using, $\tilde{J}^{x}=i \omega \sigma A_{x}^{(0)}$ where,

$$
\tilde{J}^{x}=\frac{-1}{g^{2}} \sqrt{-g} e^{-\phi} F^{u x}+\frac{1}{g_{e f f}^{2}} \sqrt{-g} A_{t}^{\prime} h_{t}^{x}
$$
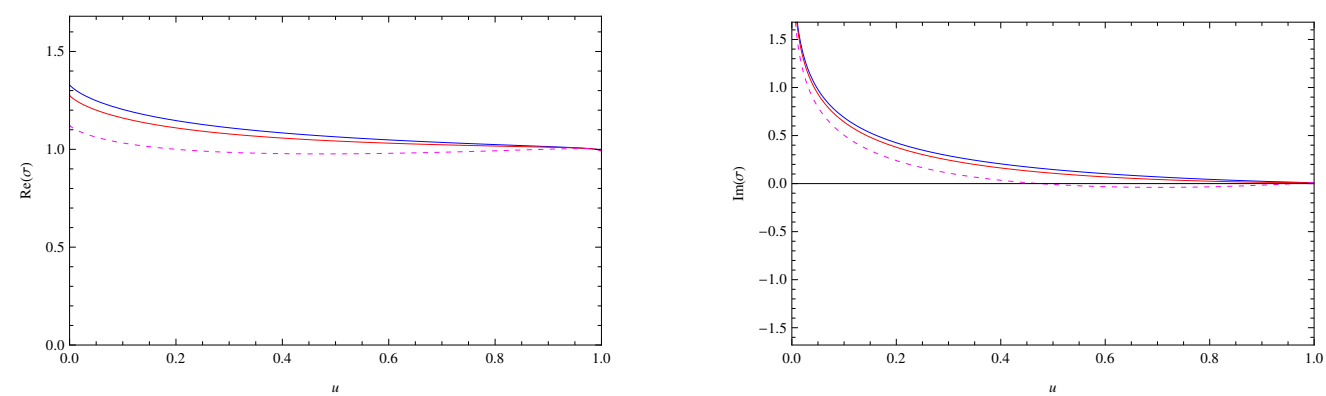

Figure 2: $\mathrm{RG}$ flow of $\mathrm{AC}$ conductivity with varying chemical potential $\mu=0.01$ (blue), 0.05 (red)and 0.1 (dashed) at $\lambda=0.05$

At horizon ac conductivity is independent of GB term and is given as,

$$
\sigma_{A}(u=1)=\frac{e^{-c}}{g^{2}} \frac{r_{+}}{l}
$$




\subsection{Diffusion Constant}

Diffusion constant can be calculated using[8],

$$
\begin{gathered}
\frac{1}{\sigma_{h}\left(u_{c}\right)}=\frac{1}{\sigma_{h}(1)}-\frac{k^{2}}{i \omega} \frac{D\left(u_{c}\right)}{\sigma_{h}(1)} \\
\sigma_{h}:=\frac{j^{z}}{f_{z t}}=\left(-u^{2} M^{\prime}\right) \frac{j^{z}}{\tilde{f}_{z t}} \\
D\left(u_{c}\right)=\frac{\frac{l^{2}}{2 r_{+}} N f\left(u_{c}\right)}{\left(2(1+a)-3 a u_{c}\right)}[1-2 \lambda(2-a)] \\
\end{gathered}
$$

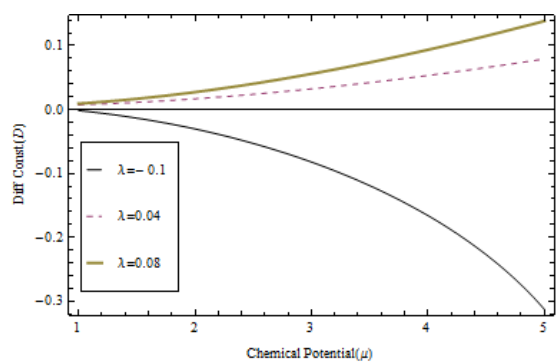

Figure 3: Dependence of diffusion constant on Gauss-Bonnet corrections with varying chemical potential

\section{Conclusions}

We have studied the behavior of ac conductivity and diffusion constant using the soft-wall model of holographic QCD. Introducing higher order gravity corrections (GB terms) makes the metric and gauge field perturbations difficult to decouple.Using the membrane paradigm approach we have calculated ac conductivity and diffusion coefficient at a cut-off. Diffusion constant shows an explicit dependence on the GB term whereas ac conductivity is independent of the GB correction at the cut-off surface. We have also found that chemical potential plays a significant role to determine the behavior of transport properties of the strongly coupled system.

\section{References}

[1] J. M. Maldacena, Int. J. Theor. Phys. 38, 1113 (1999) [Adv. Theor. Math. Phys. 2, 231 (1998)]

[2] X. H. Ge, Y. Matsuo, F. W. Shu, S. J. Sin and T. Tsukioka, Prog. Theor. Phys. 120, 833 (2008)

[3] Y. Matsuo, S. -J. Sin and Y. Zhou, JHEP 1201, 130 (2012)

[4] S. -J. Sin and Y. Zhou, JHEP 1105, 030 (2011)

[5] I. Bredberg, C. Keeler, V. Lysov and A. Strominger, JHEP 1103, 141 (2011)

[6] J. Erlich, E. Katz, D. T. Son and M. A. Stephanov, Phys. Rev. Lett. 95, 261602 (2005)

[7] A. Karch, E. Katz, D. T. Son and M. A. Stephanov, Phys. Rev. D 74, 015005 (2006)

[8] N.Bhatnagar and S. Siwach AYSS Proceeding -2016 ISBN No.978-5-9530-0416-9 